\title{
Minimization of $\boldsymbol{k}$-Variable-Mixed-Polarity Reed-Muller Expansions
}

\author{
BOGDAN J. FALKOWSKI ${ }^{\mathrm{a}, *}$ and CHIP-HONG $\mathrm{CHANG}^{\mathrm{b}, \dagger}$ \\ ${ }^{a}$ Nanyang Technological University, School of Electrical and Electronic Engineering, Blk S1, \\ Nanyang Avenue, Singapore 639798; ${ }^{\mathrm{b}}$ Nanyang Technological University, School of Electrical and Electronic \\ Engineering, Blk S2, Nanyang Avenue, Singapore 639798
}

(Received 5 June 1999; In final form 19 November 1999)

\begin{abstract}
A lookup table based method to minimize Generalized Partially-Mixed-Polarity ReedMuller (GPMPRM) expansions with $k$ mixed polarity variables has been developed. The new algorithm can produce solutions based on the desired cost criteria for the systems of completely specified functions.
\end{abstract}

Keywords: Reed-Muller expansions; Exclusive sums of products; Boolean functions

\section{INTRODUCTION}

The logic circuits using Exclusive OR (EXOR) gates are more economical than the design based on other gates for many important circuits such as adders and parity checkers. With the advent of cellular Field Programmable Gate Arrays (FPGAs) and the introduction of new Programmable Logic Devices (PLDs), for examples, Xilinx lookup based and Actel 1020 multiplexer based FPGAs, and Signetics LHS501 folded NAND devices, propagation delay and gate area is no longer a major concern in Exclusive Sum-ofproducts (ESOP) [4, 19] implementation of logic circuits. What is more, the circuits built around the EXOR gates, are easily testable [14, 15]. Fault detection of any logical circuit by verification of its Reed-Muller coefficients was considered in [14]. The upper bound on the number of ReedMuller coefficients to be verified for detection of all multiple terminal stuck-at-faults and all single input bridging faults is shown to be $n$ [14].

Unfortunately, ESOP of a Boolean function exists in many forms, and exact minimal solutions have been found practically only for functions with less than six variables [13]. Special interest and attention have been focused on two of the canonical subfamilies of ESOP, the Fixed Polarity Reed-Muller (FPRM) expansion [1,4-11, 13, 15] and the Kronecker Reed-Muller (KRM) expansion

\footnotetext{
*Corresponding author. Tel.: (65)790-4521, Fax: (65)791-2687, e-mail: efalkowski@ntu.edu.sg

${ }^{\dagger}$ Tel.: (65)790-5873, Fax: (65)792-0415, e-mail: echchang@ntu.edu.sg
} 
[19]. The former has $2^{n}$ alternative forms and the latter has $3^{n}$ alternative forms. The problem of finding optimal Reed-Muller expansions with the least number of terms received a lot of attention among the researchers. It can be reformulated in terms of coding or graph theory or solved by means of digital signal processing techniques such as fast transforms and convolution [4]. Non-exhaustive minimization methods for Reed-Muller expansions based on the extended truth vector were considered in [4]. These algorithms have a space complexity of $O\left(3^{n}\right)$. In general, the computational complexity of the minimization problem for Reed-Muller expansions seems unmanageable when the number of variables $n$ is greater than or equal to 4 . This computational complexity has led various authors to present heuristic solutions to minimization problems when $n \geq 4$ [4]. When the original function is represented in the reduced form such as arrays of cubes or decision diagrams, the methods to calculate Reed-Muller expansions directly from such representations have also been considered [1,10]. For example, in [15], an exact and quasi-minimal algorithm based on disjoint cubes has been presented. A total search is necessary to find an optimal expansion. To minimize the number of operations, such a search is performed in Gray code ordering. This algorithm can handle only a single output completely specified function. Recently a number of exact and heuristic algorithms to minimize FPRMs have been developed $[1,3,5,16,18]$. Most of the exact algorithms can minimize functions with around 15 variables. Some heuristic methods can deal with functions of about 100 input variables $[5,16]$. Owing to the high computational complexity, no exact minimization technique for canonical form more general than KRM expansion has been proposed [19]. There is another Reed-Muller canonical expansion known as Generalized ReedMuller (GRM) expansion which consists of a total of $2^{n 2^{n-1}}$ alternative forms $[2,4,8,11,19,20]$. GRM expansion can be considered as a combination of two subfamilies of Reed-Muller expansions, the Inconsistent Mixed Polarity Reed-Muller (IMPRM) expansion [2] and the FPRM expansion. Although minimal GRM expansion is expected to be closer to the minimal ESOP than the minimal KRM expansion due to the greater number of alternative forms, an exhaustive search for a minimal GRM is also computationally unfeasible even for very small number of variables $[4,11,19]$. Recently, $\mathrm{Wu}$ et al., and Zeng et al. [19,20] proposed another subfamily of GRM called the Generalized Partially-Mixed-Polarity Reed-Muller(GPMPRM) expansion. GPMPRM is a superset of FPRM which has $n 2^{n-1} 2^{2^{n-1}}-(n-1) 2^{n}$ alternative forms. Based on the number of alternative forms, it is believed that the minimal GPMPRM expansion is still much closer to the minimal ESOP than the minimal KRM expansion.

The definition of GPMPRM expansion from [19] with only one mixed polarity variable was extended to $k$ mixed polarity variables in $[9,20]$. It should be stressed, however that the authors of [20] have not found an efficient exact algorithm for $k>1$, the task which is solved in the current paper. The extension to $k$ mixed polarity variables further reduces the gap between the minimal GPMPRM and the minimal ESOP since the total number of alternative forms is ${ }^{n} C_{k} 2^{n-k} 2^{k 2^{n-1}}-\left({ }^{n} C_{k}-1\right) 2^{n}$ based on the new definition. The lookup table based approach for the exact minimization of FPRM expansion developed by the authors in $[1,7]$ is modified to generate a minimal GPMPRM expansion of $k$ mixed polarity variables. Contrary to all algorithms known from the literature $[12,19,20]$, our algorithm for the minimization of GPMPRM expansions, is adaptable to different cost criteria, for instance, the total number of unique products, the total number of unique literals and the linear combination of both criteria. Based on the size of the tackled problem, our algorithm can use different size of the lookup table to trade the space complexity problem into the processing time complexity problem. Experimental results show that even without considering all possible combinations of $k$ variables as the mixed polarity variables, for most functions reported in [19], which considered all combinations of one 
mixed polarity variable, the quality of the results obtained by our algorithm is either the same or better.

\section{BASIC DEFINITIONS}

An $n$-variable Boolean function can be expressed as a canonical Reed-Muller expansion [1-11, 14-20] of $2^{n}$ terms as follows:

$$
F\left(x_{n}, x_{n-1}, \ldots, x_{1}\right)=\bigoplus_{j=0}^{2^{n}-1} a_{j} \prod_{i=1}^{n} \dot{x}_{i}^{j_{i}}
$$

where $\oplus$ denotes the modulo- 2 addition, $a_{j} \in(0,1)$ is called a Reed-Muller coefficient and $j_{i} \in(0,1)$ is called the power of $\dot{x}_{i}$ such that $\left\langle j_{n} j_{n-1} \cdots j_{2} j_{1}\right\rangle$ is equal to the binary representation of $j$. When $j_{i}=0$, the literal $\dot{x}_{i}$ is absent in the product term $\prod_{j=1}^{n} \dot{x}_{i}^{j_{i}}$, otherwise it is present in the product term. When each literal $\left(\dot{x}_{i}, \quad i=1,2, \ldots, n\right)$ throughout the expression (1) assumes either true or complemented form but not both forms simultaneously, such an expression is known as a Fixed Polarity Reed-Muller (FPRM) expansion $[1,4-11,13,15]$. If all the literals in (1) can assume either complemented or non-complemented form in any combination, it is known as the Generalized Reed-Muller $(G R M)$ expansion [2, 4, 11, 19]. Since there are $n 2^{n-1}$ literals in the complete expression (1), there are $2^{n 2^{n-1}}$ possible GRM expansions, including $2^{n}$ FPRM expansions.

In [19], a strong constraint is placed in the definition of GRM expansion to obtain the Generalized Partially-Mixed-Polarity Reed-Muller (GPMPRM) expansion. It is a subset of GRM expansions that encloses the FPRM expansions. However, the requirement that the polarities of all but one variable have to be fixed can be relaxed. A more general definition of GPMPRM expansion, the possibility which was mentioned in $[9,20]$ is given as follows:

Definition 1 The Generalized Partially-MixedPolarity Reed-Muller (GPMPRM) expansions are obtained by allowing the $k 2^{n-1}$ literals of $k$ variables in expression (1) to freely assume either polarity while maintaining consistent fixed polarities for all the literals of the remaining variables.

Under this new definition, for an $n$-variable completely specified Boolean function, there are ${ }^{n} C_{k} 2^{n-k} 2^{2^{n-1}}-\left({ }^{n} C_{k}-1\right) 2^{n}$ alternative GPMPRM forms. The proof of this fact can be constructed in a similar way as that shown in [19].

Let $x_{i}^{\omega_{i}, j_{i}}$ denote $\bar{x}_{i}, x_{i}$ or 1 for $\left(\omega_{i}, j_{i}\right)=(1,1)$, $(0,1)$ and $(-, 0)$ respectively where the symbol '-, stands for either 0 or 1 . A GPMPRM product term can be expressed as $\prod_{i=1}^{n} x_{i}^{\omega_{i}, j_{i}}$ where the decimal numbers $j=\sum_{i=1}^{n} j_{i} \times 2^{i-1}$ and $\omega=$ $\sum_{i=1}^{n} \omega_{i} \times 2^{i-1}$ are indicators of the appearances and polarities of the literals in the product term. Each GPMPRM product term has a polarity number, denoted by $\omega$ or $\left\langle\omega_{n} \omega_{n-1} \cdots \omega_{2} \omega_{1}\right\rangle$, which is a binary $n$-tuple formed by writing a 0 or a 1 for each literal according to whether the literal is used in a positive or negative form. It is more convenient to treat the literals present in a GPMPRM product term as a conjunction of two parts: the fixed polarity part $\prod_{i=k+1}^{n} x_{i}^{\omega_{1}, j_{l}}$ and the mixed polarity part $\prod_{i=1}^{k} x_{i}^{\omega_{i}, j_{i}}$. Since the $(n-k)$ digits of the polarity number $\omega$ are identical for all product terms of a GPMPRM expansion with $k$ mixed polarity variables, only $k$ digits of $\omega$ corresponding to the mixed polarity variables need to be specified separately for every product term.

Definition 2 A polarity vector $A^{\omega}$ is an ordered set of all $2^{n}$ FPRM expansion coefficients $\left[a_{0} a_{1} \cdots a_{2^{n}-1}\right]$ in some chosen polarity number $\omega$. $A^{\omega}$ can also be represented by a decimal number $\sum_{i=0}^{2^{n}-1} a_{i} \times 2^{i}$ with $a_{0}$ as the least significant bit and $a_{2^{n}-1}$ the most significant bit of its binary equivalent.

Definition 3 The polarity coefficient matrix $P C(F)[1,7,10]$ of an $n$-variable Boolean function $F$ is a $2^{n} \times 2^{n}$ binary matrix, where every row corresponds to a polarity vector $A^{\omega}$ in a different polarity number $\omega$. 
Every element $m_{i j}$ (row $i$, column $j$ ) of the polarity coefficient matrix $P C(F)$ describes the coefficient $a_{j}$ of the FPRM expansion with polarity $\omega=i$.

DEFINITION 4 The Hamming weight of an integer $i$, denoted by $H(i)$ is the number of ' 1 ' in its binary representation.

\section{MINIMIZATION OF GPMPRM EXPANSIONS}

Recently, an algorithm has been developed that utilizes only a subset of Walsh coefficients to reveal all the information carried by the polarity coefficient matrix of any three variable Boolean functions [6]. Each class of the functions is associated with a specific subroutine that computes the optimal polarities, optimal weights, optimal fixed polarity Reed-Muller expansions etc., without resorting to an exhaustive search. Direct extension of the method in [6] to handle larger Boolean functions with the number of variables $n>3$ is unmanageable due to the increasing number of different classes. Nevertheless, exact optimal generation of FPRM expansions for large $n$ have been solved by reducing the polarity coefficient matrix into submatrices of smaller dimension such that each submatrix is a polarity coefficient matrix of a subfunction obtained by either Shannon's decomposition or Boolean difference with respect to some variables [1,7]. Similar approach as [1] can be applied to the minimization of GPMPRM expansions by selecting an optimal FPRM expansion for each subfunction, with the exception that the $k$ mixed polarity variables may have different polarities for different subfunctions.

Lemma 1 The polarity coefficient matrix $P C(F)$ of an n-variable completely specified Boolean function $F(X)$, can be partitioned into four submatrices of order $2^{n-1}$ as $[1,8-10]$ :

$$
P C(F)=\left\lceil\begin{array}{l}
P C\left(f^{0}\right) P C\left(f^{0} \oplus f^{1}\right) \\
P C\left(f^{1}\right) P C\left(f^{0} \oplus f^{1}\right)
\end{array}\right\rceil
$$

where $f^{0}$ and $f^{1}$ are cofactors of Shannon's decomposition of $F(X)$ evaluated at $x_{n}=0$ and $x_{n}=1$, respectively.

A subfunction $f^{i}, i=0,1,2$ for an $n$-variable Boolean function $F$ is defined as:

$$
\begin{aligned}
& f^{0}=F\left(0, x_{n-1}, \ldots, x_{2}, x_{1}\right) \\
& f^{1}=F\left(1, x_{n-1}, \ldots, x_{2}, x_{1}\right) \\
& f^{2}=f^{0} \oplus f^{1}
\end{aligned}
$$

By applying (2) recursively, the polarity coefficient matrix of order $2^{n}$ can be partitioned into $p^{2}$ submatrices of order $2^{k}$, where $p=2^{n-k}$. Each submatrix is a polarity coefficient matrix of a $k$-variable subfunction and the total number of unique subfunctions is equal to $3^{n-k}$.

It should be noted that the above decomposition into subfunctions does not have to begin with the most significant variable $x_{n}$. In general, a subfunction of $k$ variables, $1 \leq k<n$, can be formed by decomposing or taking the Boolean difference of $F$ with respect to some $n-k$ variables. Each subfunction $f^{\alpha}$ of $k$ variables is associated with a ternary $(n-k)$-tuple $\left\langle\alpha_{n-k} \cdots \alpha_{2} \alpha_{1}\right\rangle$ where $\alpha_{i} \in$ $(0,1,2)$. If $\alpha_{i} \in(0,1)$ for all $1 \leq i \leq n-k, \alpha$ is said to be reducible to a binary $(n-k)$-tuple and $f^{\alpha}$ is a cofactor formed from $F$ by substituting all the $k$ variables with $\alpha_{i}$ in the following manner: $x_{n}$ with $\alpha_{n-k}, x_{n-1}$ with $\alpha_{n-k-1}$, etc. If there exists an $\alpha_{i}=2$ for any $1 \leq i \leq(n-k)$, then $f^{\alpha}=$ $f^{\beta} \oplus f^{\gamma}$ where $\beta=\left\langle\alpha_{n-k} \cdots \alpha_{i+1} 0 \alpha_{i-1} \cdots \alpha_{2} \alpha_{1}\right\rangle$ and $\gamma=\left\langle\alpha_{n-k} \cdots \alpha_{i+1} 1 \alpha_{i-1} \cdots \alpha_{2} \alpha_{1}\right\rangle$. It is obvious that two or more different $\alpha_{i}, \alpha_{j}$, etc., $i \neq j$, can be equal to 2 in $f^{\alpha}$.

For simplicity, in the sequel, we assume that the $k$ mixed polarity variables of a GPMPRM expansion are $x_{1}, x_{2}, \ldots, x_{k}$ and the fixed polarity variables are $x_{k+1}, x_{k+2}, \ldots, x_{n}$. A different set of mixed polarity variable affects only the indexing of the variables which can be corrected by simply renaming the indices of the affected variables or reordering the input variables of the function. Explicitly, we use $r=\left\langle\omega_{n} \omega_{n-1} \cdots \omega_{k+1}\right\rangle$ to denote the fixed polarity number for the set of $(n-k)$ fixed polarity variables and $\phi_{j}=\left\langle\omega_{k} \omega_{k-1} \cdots \omega_{1}\right\rangle$ to denote the mixed 
polarity number for the $k$ mixed polarity variables of the $q$-th GPMPRM product term where $j 2^{k} \leq q<(j+1) 2^{k}$ and $0 \leq j \leq 2^{n-k}-1$.

Theorem 1 For an $n$-variable Boolean function $F$, the minimal number of product terms, $w_{p}(F, r)$ of a GPMPRM expansion with fixed polarity number $r$ is given by:

$$
w_{p}(F, r)=\sum_{j=0}^{2^{n-k}-1} w_{p}\left(f^{r * j}, \phi_{j}\right)
$$

where $1 \leq k<n$, and $j$ is the decimal equivalent of binary $(n-k)$-tuple $\left\langle j_{n-k} j_{n-k-1} \cdots j_{1}\right\rangle \cdot w_{p}\left(f^{r * j}, \phi_{j}\right)$ is the number of product terms of the optimal FPRM expansion of $k$-variable subfunction $f^{r * j}$ with polarity $\phi_{j}=\left\langle\omega_{k} \omega_{k-1} \cdots \omega_{1}\right\rangle$.

The operator ${ }^{*}:\{0,1\}^{k} \times\{0,1\}^{k} \rightarrow\{0,1,2\}^{k}$ is defined by the following componentwise table of operation:

second operand

first

operand

\begin{tabular}{|c|c|c|}
\multicolumn{3}{|c|}{ second operand } \\
\hline$*$ & 0 & 1 \\
\hline 0 & 0 & 2 \\
\hline 1 & 1 & 2 \\
\hline
\end{tabular}

Proof Let $w_{p}(F, \omega)$ denote the number of product terms of the FPRM expansion of $F$ in polarity $\omega$. The weight vector $W_{p}(F)=\left[w_{p}(F, 0) w_{p}(F, 1) \cdots w_{p}\right.$ $\left.\left(F, 2^{n-1}\right)\right]^{\mathrm{T}}$ is an ordered set of the number of product terms in each row of $P C(F)$ for an $n$ variable Boolean function $F . W_{p}(F)$ can be obtained in a similar recursive way as Lemma 1 from the weight vectors $W\left(f^{0}\right), W\left(f^{1}\right)$ and $W\left(f^{0} \oplus f^{1}\right)$ corresponding to the FPRM expansions of subfunctions $f^{0}, f^{1}$ and $f^{2}$ respectively.

$$
\begin{aligned}
& W_{p}(F)=\left[\begin{array}{l}
W_{p}\left(f^{0}\right)+W_{p}\left(f^{2}\right) \\
W_{p}\left(f^{1}\right)+W_{p}\left(f^{2}\right)
\end{array}\right]= \\
& {\left[\begin{array}{l}
W_{p}\left(f^{00}\right)+W_{p}\left(f^{02}\right)+W_{p}\left(f^{20}\right)+W_{p}\left(f^{22}\right) \\
W_{p}\left(f^{01}\right)+W_{p}\left(f^{02}\right)+W_{p}\left(f^{21}\right)+W_{p}\left(f^{22}\right) \\
W_{p}\left(f^{10}\right)+W_{p}\left(f^{12}\right)+W_{p}\left(f^{20}\right)+W_{p}\left(f^{22}\right) \\
W_{p}\left(f^{11}\right)+W_{p}\left(f^{12}\right)+W_{p}\left(f^{21}\right)+W_{p}\left(f^{22}\right)
\end{array}\right]} \\
& =\ldots \text { etc. }
\end{aligned}
$$

After $n-k$ recursions, there are $2^{n-k}$ rows each having $2^{n-k}$ weight vectors $W_{p}$ to be summed. The first weight vector in the $r$-th $\left(r=0,1, \ldots, 2^{n-k}-1\right)$ row is the weight vector $W_{p}$ of the cofactor generated by decomposing $F$ with respect to $\prod_{i=1}^{n-k} x_{k+i}^{r_{1}, 1}$. If the cofactor at $r$-th row is denoted by $f^{r}$, the subfunction appearing in the $j$-th $\left(j=0,1, \ldots, 2^{n-k}-1\right)$ weight vector $W_{p}$ of the same row is given by $f^{r * j}$. If the decomposition of $F$ is performed over the set of fixed polarity literals with polarity number $r$, the subfunctions $f^{r * j}$ for all $j=0,1, \ldots, 2^{n-k}-1$ are Boolean functions of $k$ mixed polarity variables. Since the $k$ variables of the subfunction $f^{r * j}$ are allowed to have mixed polarities in a GPMPRM expansion, the best weight (smallest number of product terms) with polarity number $\phi_{j}$ of $W_{p}\left(f^{r * j}\right)$ for each $j$ is selected. Hence (3) is obtained.

THEOREM 2 The minimal number of literals, $w_{l}(F, r)$ of a GPMPRM expansion with fixed polarity number $r$ is given by:

$$
w_{l}(F, r)=\sum_{j=0}^{2^{n-k}-1}\left[w_{l}\left(f^{r * j}, \phi_{j}\right)+H(j) \times w_{p}\left(f^{r * j}, \phi_{j}\right)\right]
$$

where the symbols $j, k, r$ and the operator * have been defined earlier. Here, $\phi_{j}$ refers to the polarity number of the FPRM expansion of $k$-variables subfunction $f^{r * j}$ that has the smallest number of literals, with priority given to FPRM expansion with $a_{0}=0$ when there is a tie. $w_{l}\left(f^{r * j}, \phi_{j}\right)$ and $w_{p}\left(f^{r * j}, \phi_{j}\right)$ are the number of literals and product terms of the FPRM expansion of $f^{r * j}$ in polarity $\phi_{j}$ respectively. $H(j)$ is the Hamming weight of the integer $j$.

Proof When the polarity coefficient matrix (2) is expanded recursively $n-k$ times, the submatrix at the $r$-th row and $j$-th column of $P C(F)$ corresponds to a polarity coefficient matrix of a subfunction $f^{r * j}$. Each such submatrix $P C\left(f^{r * j}\right)$ has the dimension of $2^{k} \times 2^{k}$. If $\phi_{j}$ is the row number of $P C\left(f^{r * j}\right)$ that has the smallest number of literals, each non zero 
entry at row $\phi_{j}$ and column $g$ of the submatrix $P C\left(f^{r * j}\right)$ contributes to the GPMPRM expansion a product term $\prod_{i=k+1}^{n} x_{i}^{r_{i-k}, j_{i-k}} \cdot \prod_{i=1}^{k} x_{i}^{\phi_{j}, g_{i}}$. The number of literals in the product $\prod_{i=1}^{k} x_{i}^{\phi_{j}, g_{i}}$ is equal to $w_{l}\left(f^{r * j}, \phi_{j}\right)$. Since there are $H(j)$ additional fixed polarity literals in the product $\prod_{i=k+1}^{n} x_{i}^{r_{l-k}, j_{i-k}}$, the total number of literals contributed by any submatrix $P C\left(f^{r * j}\right)$ is given by $w_{l}\left(f^{r * j}, \phi_{j}\right)+$ $H(j) w_{p}\left(f^{r * j}, \phi_{j}\right)$. Hence (4) follows.

For a small number of mixed polarity variables $k$, typically $k \leq 5$, instead of searching for the optimal polarity $\phi_{j}$ and then calculating $w_{p}\left(f^{r * j}, \phi_{j}\right)$ and $w_{l}\left(f^{r * j}, \phi_{j}\right)$ from $P C\left(f^{r * j}\right)$, lookup table can be used. For the minimization of the number of product terms in the GPMPRM expansion, each record of the lookup table contains an optimal polarity $\phi_{j}$, the polarity vector $A^{\phi_{j}}$ and its number of product terms $w_{p}\left(f^{\alpha}, \phi_{j}\right)$ for the subfunction $f^{\alpha}$ where $\alpha=r * j$. For minimization with respect to the number of literals, the record for the subfunction $f^{\alpha}$ consists of the polarity $\phi_{j}$ for minimal number of literals, the polarity vector $A^{\phi_{j}}$, and its number of literals $w_{l}\left(f^{\alpha}, \phi_{j}\right)$ and product terms $w_{p}\left(f^{\alpha}, \phi_{j}\right)$. In order to use the $k$-variable subfunction $f^{\alpha}$ as an index to the lookup table, we assign to $f^{\alpha}$ a unique integer value $\mathfrak{R}\left(f^{\alpha}\right)$ whose binary equivalent is $\left\langle m_{2^{k}-1} m_{2^{k}-2} \cdots m_{1} m_{0}\right\rangle$ where $m_{i} \in(0,1)$ is the value of the $i$-th minterm of $f^{\alpha}$. Clearly, $\mathfrak{R}\left(\overline{f^{\alpha}}\right)=$ $2^{2^{k}}-1-\mathfrak{R}\left(f^{\alpha}\right) \quad$ and $\quad \mathfrak{R}(f \oplus g)=\mathfrak{R}(f) \oplus_{\mathrm{d}} \mathfrak{R}(g)$ where the symbol ' $\oplus_{\mathrm{d}}$ ' is the dyadic addition or the addition of two integers over $G F(2)$. The polarity vector field in each record is also represented by an integer whose binary representation is $\left\langle a_{7} a_{6} \cdots a_{1} a_{0}\right\rangle$ where $a_{i}$ is the coefficient of the FPRM expansion of the subfunction $f^{\alpha}$ in the selected optimal polarity.

As $A^{\phi_{j}}\left(\overline{f^{\alpha}}\right)$ and $A^{\phi_{j}}\left(f^{\alpha}\right)$ differ only in the constant term $a_{0}, w_{l}\left(\overline{f^{\alpha}}, \phi_{j}\right)=w_{l}\left(f^{\alpha}, \phi_{j}\right)$. However, from Theorem 2, the constant term of each subfunction contributes $H(j)$ literals to the final GPMPRM expansion and can not be neglected. When seeking for the minimal number of literals in the lookup table, if there are more than one optimal polarities for a subfunction, the optimal polarity for the polarity vector with $a_{0}=0$ is chosen.

Based on Theorems 1 and 2, the algorithm for the fast computation of the minimal GPMPRM expansion is given in Figure 1.

In Figure 1, Tables I and II are lookup tables for different options of minimization specified by the Boolean variable optimize_nof_products. Each product term of a GPMPRM is considered as a concatenation of two products, i.e., the products of the fixed polarity variables and the mixed polarity variables. The presence of a mixed polarity product is indicated by a ' 1 ' in the binary $k$-tuple of $o p t_{-} G P M P R M[j]$ while its associated fixed polarity variables is indicated by the ' 1 's in the binary $(n-k)$ tuple of $j$. The polarities of the fixed polarity variables of the final GPMPRM expansion are stored in the bit fields of the variable optimal_fixed_polarity, and the polarities of the mixed polarity variables in the products terms of opt_GPMPRM[j] are stored in the bit fields of opt_mixed_polarity $[j]$.

If we consider only one arbitrary set of $k$ mixed polarity variables, the outer loop of GPMPRM can be removed and the resulting GPMPRM expansion is optimum with respect to a given set of mixed polarity variables. Such a constraint is frequently encountered in practice as it may be more cost effective to restrict the privilege of dual polarities to only some specific variables.

Example 1 Consider the 5-variable Boolean function $F\left(x_{5}, x_{4}, x_{3}, x_{2}, x_{1}\right)=\sum m(8,10,11,16,17$, $19,23,24,26,27)$. For $k=3$, there are ${ }^{5} C_{3}=10$ possible selections of three mixed polarity variables. For simplicity, we consider only the case of $x_{3}, x_{2}$ and $x_{1}$ as the set of mixed polarity variables. The number of Reed-Muller products for each fixed polarity number $r=0,1,2$ and 3 are determined according to the algorithm in Figure 1 as follows:

For $r=0, f^{00 * 00}=f^{00}=\langle 00000000\rangle ; f^{00 * 01}=f^{02}=$ $f^{00} \oplus_{\mathrm{d}} f^{01}=\langle 00000000\rangle \oplus_{\mathrm{d}}\langle 00001101\rangle=\langle 00001101\rangle$; $f^{00 * 10}=f^{20}=f^{00} \oplus_{\mathrm{d}} f^{10}=\langle 00000000\rangle \oplus_{\mathrm{d}}$ $\langle 10001011\rangle=\langle 10001011\rangle ; \quad f^{00 * 11}=f^{22}=f^{20} \oplus_{\mathrm{d}}$ 


\section{G PM PRM}

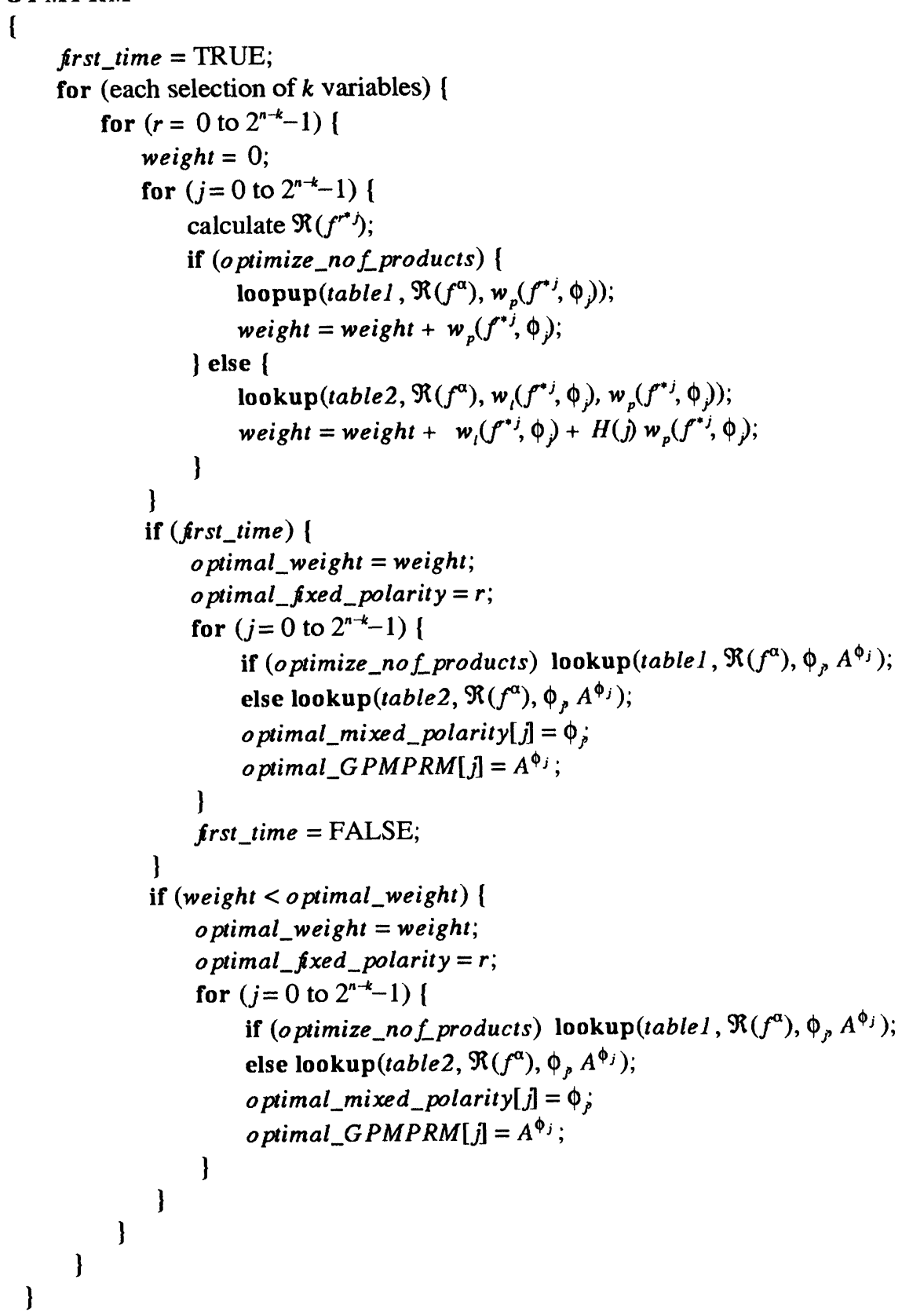

FIGURE 1 Generation of minimal GPMPRM expansion.

\begin{tabular}{|c|c|}
\hline$f^{21}=f^{20} \oplus_{\mathrm{d}} f^{01} \oplus_{\mathrm{d}} f^{11}=\langle 10001011\rangle \oplus_{\mathrm{d}}\langle 00001101\rangle \oplus_{\mathrm{d}}$ & Reed-Muller product terms for the subfunctions \\
\hline $\begin{array}{l}\langle 00001101\rangle=\langle 10001011\rangle \\
\mathfrak{R}(\langle 00001101))=13 \text { and }\end{array}$ & $f^{\alpha}$. From the lookup table, $w_{p}(0)=0, w_{p}(13)=$ \\
\hline $\begin{array}{l}101\rangle)=13 \text { and } 9 \\
\left.\left(f^{\alpha}\right)\right) \text { denote the }\end{array}$ & $w_{p}(13)+w_{p}(139)+w_{p}(139)=$ \\
\hline
\end{tabular}


TABLE I Benchmark results for GPMPRM and FPRM

\begin{tabular}{|c|c|c|c|c|c|c|c|c|}
\hline \multirow[b]{2}{*}{ Function } & \multirow[b]{2}{*}{$n$} & \multirow[b]{2}{*}{$m$} & \multicolumn{2}{|c|}{ GPMPRM } & \multicolumn{2}{|c|}{ FPRM } & \multicolumn{2}{|c|}{ Time (sec) } \\
\hline & & & $w_{p}$ & $w_{l}$ & $w_{p}$ & $w_{l}$ & usr & sys \\
\hline $5 x p 1$ & 7 & 10 & 59 & 198 & 61 & 224 & 5.13 & 0.02 \\
\hline 9sym & 9 & 1 & 136 & 504 & 173 & 636 & 34.14 & 0.01 \\
\hline conl & 7 & 2 & 13 & 37 & 17 & 48 & 0.21 & 0.03 \\
\hline $\operatorname{misex} 1$ & 8 & 7 & 16 & 51 & 20 & 68 & 7.91 & 0.01 \\
\hline $\mathrm{rd} 53$ & 5 & 3 & 20 & 45 & 20 & 45 & 0.02 & 0.03 \\
\hline $\mathrm{rd} 73$ & 7 & 3 & 63 & 189 & 63 & 189 & 2.16 & 0.02 \\
\hline $\mathrm{rd} 84$ & 8 & 4 & 107 & 352 & 107 & 352 & 31.00 & 0.02 \\
\hline sao2 & 10 & 4 & 70 & 372 & 100 & 707 & 308.46 & 0.06 \\
\hline squar5 & 5 & 8 & 23 & 56 & 23 & 56 & 0.10 & 0.03 \\
\hline xor5 & 5 & 1 & 5 & 5 & 5 & 5 & 0.01 & 0.01 \\
\hline Z9sym & 9 & 1 & 136 & 504 & 173 & 636 & 126.87 & 0.02 \\
\hline clip & 9 & 5 & 173 & 757 & 206 & 995 & 138.11 & 0.05 \\
\hline
\end{tabular}

TABLE II Benchmark results of single output for GPMPRM, GPMP and CGRMIN

\begin{tabular}{|c|c|c|c|c|c|c|c|c|}
\hline \multirow[b]{2}{*}{ Function } & \multirow[b]{2}{*}{$n$} & \multicolumn{3}{|c|}{ GPMPRM } & \multicolumn{2}{|c|}{ GPMP } & \multicolumn{2}{|c|}{ CGRMIN } \\
\hline & & $w_{p}$ & $w_{l}$ & Time & $w_{p}$ & Time & $w_{p}$ & Time \\
\hline $5 x p 11$ & 7 & 9 & 35 & 0.02 & 9 & 0.22 & 12 & - \\
\hline $5 x p 13$ & 7 & 16 & 47 & 0.03 & 14 & 0.22 & 19 & - \\
\hline $5 x p 15$ & 7 & 7 & 13 & 0.02 & 6 & 0.20 & 7 & - \\
\hline 9sym & 9 & 136 & 504 & 0.01 & 139 & 3.9 & 173 & $1,851.3$ \\
\hline $\operatorname{con} 12$ & 7 & 5 & 12 & 0.02 & 9 & 0.22 & 12 & 1.2 \\
\hline rd532 & 5 & 5 & 5 & 0.01 & 5 & 0.03 & 5 & 1.1 \\
\hline rd732 & 7 & 7 & 7 & 0.02 & 7 & 0.22 & 7 & 49.6 \\
\hline rd842 & 8 & 8 & 8 & 0.02 & 8 & 0.83 & 8 & 364.2 \\
\hline xor5 & 5 & 5 & 5 & 0.01 & 5 & 0.00 & 5 & - \\
\hline sao 22 & 10 & 38 & 182 & 0.02 & 37 & 16.58 & 58 & 642.1 \\
\hline sao 23 & 10 & 32 & 158 & 0.02 & 35 & 16.52 & 47 & 905.9 \\
\hline $\mathrm{z} 4 \mathrm{ml}$ & 7 & 15 & 56 & 0.02 & 22 & 0.2 & 25 & - \\
\hline
\end{tabular}

'-': data not available.

For $r=1, f^{01 * 00}=f^{01}=\langle 00001101\rangle ; f^{01 * 01}=$ $f^{02}=f^{00} \oplus_{\mathrm{d}} f^{01}=\langle 00000000\rangle \oplus_{\mathrm{d}}\langle 00001101\rangle=$ $\langle 00001101\rangle ; f^{01 * 10}=f^{21}=f^{01} \oplus_{\mathrm{d}} f^{11}=\langle 00001101\rangle \oplus_{\mathrm{d}}$ $\langle 00001101\rangle=\langle 00000000\rangle ; \quad f^{01 * 11}=f^{22}=$ $\langle 10001011\rangle$. From $(3), w(F, 1)=w_{p}(13)+w_{p}(13)+$ $w_{p}(0)+w_{p}(139)=2+2+0+3=7$.

For $r=2, f^{10 * 00}=f^{10}=\langle 10001011\rangle ; f^{10 * 01}=$ $f^{12}=f^{10} \oplus_{\mathrm{d}} f^{11}=\langle 10001011\rangle \oplus_{\mathrm{d}}\langle 00001101\rangle=$ $\langle 10000110\rangle ; f^{10 * 10}=f^{20}=\langle 10001011\rangle ; f^{10 * 11}=$ $f^{22}=\langle 10001011\rangle . \mathfrak{R}(\langle 10000110\rangle)=134$. From (3), $w(F, 2)=w_{p}(139)+w_{p}(134)+w_{p}(139)+w_{p}(139)=$ $3+4+3+3=13$.

For $r=3, f^{11 * 00}=f^{11}=\langle 00001101\rangle ; f^{11 * 01}=$ $f^{12}=\langle 10000110\rangle ; f^{11 * 10}=f^{21}=\langle 00000000\rangle$; $f^{11 * 11}=f^{22}=\langle 10001011\rangle . \quad$ From $(3), \quad w(F, 3)=$ $w_{p}(13)+w_{p}(134)+w_{p}(0)+w_{p}(139)=2+4+0+$ $3=9$.

Since $w(F, 1)=7$ is the minimal weight, the fixed polarity literals are chosen to be $x_{5}$ and $\bar{x}_{4}$. Let $\phi\left(\Re\left(f^{\alpha}\right)\right)$ and $A^{\phi_{J}}\left(\mathfrak{R}\left(f^{\alpha}\right)\right)$ denote the optimal polarity number and FPRM expansion for the subfunctions $f^{\alpha}$ obtained from the lookup table, respectively. Then, $\phi_{0}=\phi_{1}=\phi(13)=6$, $\phi_{2}=\phi(0)=0$ and $\phi_{3}=\phi(139)=4$. Also, $A^{6}(13)=$ $144=\langle 10010000\rangle=\bar{x}_{3} \oplus \bar{x}_{3} \bar{x}_{2} x_{1}, \quad A^{0}(0)=0=$ $\langle 00000000\rangle$ and $A^{4}(139)=88=\langle 01011000\rangle=$ $x_{2} x_{1} \oplus \bar{x}_{3} \oplus \bar{x}_{3} x_{2}$. Hence, the GPMPRM expansion of $F$ is given by $\left(\bar{x}_{3} \oplus \bar{x}_{3} \bar{x}_{2} x_{1}\right) \oplus \bar{x}_{4}\left(\bar{x}_{3} \oplus \bar{x}_{3} \bar{x}_{2} x_{1}\right)$ 
$\oplus x_{5} \bar{x}_{4}\left(x_{2} x_{1} \oplus \bar{x}_{3} \oplus \bar{x}_{3} x_{2}\right)=\bar{x}_{3} \oplus \bar{x}_{3} \bar{x}_{2} x_{1} \oplus \bar{x}_{4} \bar{x}_{3} \oplus$ $\bar{x}_{4} \bar{x}_{3} \bar{x}_{2} x_{1} \oplus x_{5} \bar{x}_{4} x_{2} x_{1} \oplus x_{5} \bar{x}_{4} \bar{x}_{3} \oplus x_{5} \bar{x}_{4} \bar{x}_{3} x_{2}$.

\section{EXPERIMENTAL RESULTS}

The new algorithm is implemented on the HP Apollo Series 715 workstation with $k=3$ mixed polarity variables. The current implementation can calculate the minimal GPMPRM expansions based on either the minimal number of products, $w_{p}$ or literals, $w_{l}$ although theoretically, any cost function of the form $a w_{l}+b w_{p}$ is possible where $a$ and $b$ are integer constants. The quality of the results for a minimal GPMPRM expansion with three mixed polarity variables always outperforms the procedure with only one mixed polarity variable. For better analysis and comparison with the fast algorithm of [19], an arbitrary set of three mixed polarity variables is selected instead of cycling through all possible selections of three variables to seek for the exact optimal GPMPRM. A range of benchmark examples have been tested. Some multiple output two level examples in pla format from MCNC benchmarks minimized with $w_{p}$ and $w_{l}$ as cost functions are compared in Table I with the results obtained from the exact FPRM minimizers $[1,3]$. The column labeled Time is the user (usr) and system (sys) execution time of our current implementation in second. For most functions, our results are significantly better than the exact FPRM expansions, particularly in terms of the number of literals. It should be noted that the savings in the total number of literals for GPMPRM expansion is more prominent than the savings in the number of product terms. Table II summarizes the comparison between the quality and system execution time of our algorithm and those of the exact minimizer with all possible selections of one mixed polarity variable, GPMP [19] and exact FPRM minimizer, CGRMIN [15] for single output functions. Despite the simplification of using an arbitrary set of three mixed polarity variables, our results for majority of the functions are either the same or better than that for GPMP and outperform CGRMIN. Moreover, the processing time of our algorithm is remarkably lower than GPMP and CGRMIN. It should be however noticed, that Table II uses time taken from $[15,19]$ directly so the time is also influenced by the different workstations used in each of the experiments.

\section{CONCLUSION}

This paper solves the open problem stated in [20] on how to minimize GPMPRM expansions with $k$ mixed polarity variables. For such a case, this expansion has ${ }^{n} C_{k} 2^{n-k} 2^{k 2^{n-1}}-\left({ }^{n} C_{k}-1\right) 2^{n}$ alternative forms which is closer to the ESOP than the original definition. An efficient lookup table based method is presented in this paper for the heuristic minimization of GPMPRM expansions with $k<6$ mixed polarity variables for multiple output functions. Due to the inherent nature of NP problem, the presented algorithm is highly efficient for up to ten input variables with $k=3$. It is also adaptable to various cost functions which is what lacks in the existing minimizer $[19,20]$.

\section{References}

[1] Chang, C. H. and Falkowski, B. J., "Flexible optimization of fixed polarity Reed-Muller expansions for multiple output completely and incompletely specified Boolean functions", In: Proc. IEEE/IEICE Asia and South Pacific Design Automation Conf. (ASP-DAC '95), Makuhari, Chiba, Japan, pp. 335-340, Aug., 1995.

[2] Cohn, M. (1962). "Inconsistent canonical forms of switching functions", IRE Trans. Electronic Computers, 11, 284-285,

[3] Csanky, L., Perkowski, M. A. and Schaefer, I., "Canonical restricted mixed-polarity exclusive-OR sums of products and the efficient algorithm for their minimization", IEE Proc., Pt. E, 140(1), 69-77, Jan., 1993.

[4] Davio, P., Deschamps, J. P. and Thayse, A., Discrete and Switching Functions. New York: McGraw-Hill, 1978.

[5] Drechsler, R., Theobald, M. and Becker, B., "Fast OFDD-based minimization of fixed polarity Reed-Muller expressions", IEEE Trans. Comput., 45(11), 1294-1299, Nov., 1996.

[6] Falkowski, B. J. and Chang, C. H.,"Generation of fixed polarity Reed-Muller expansions from subset of Walsh spectral coefficients for completely specified Boolean functions", In: Proc. of 5th Workshop on Spectral Techniques, Beijing, China, pp. 214-219, Mar., 1994. 
[7] Falkowski, B. J. and Chang, C. H. (1995). "An exact minimizer of fixed polarity Reed-Muller expansions", Int. J. Electronics, 79(4), 389-409.

[8] Falkowski, B. J. and Chang, C. H., "Minimization of partially-mixed-polarity Reed-Muller expansions for multiple output incompletely specified Boolean functions", In: Proc. of 6th Workshop on Post Binary Ultra-Large Scale Integration, Antigonish, Nova Scotia, Canada, May, 1997.

[9] Falkowski, B. J. and Chang, C. H., "Optimization of partially-mixed-polarity Reed-Muller expansions", In: Proc. of IEEE Symposium on Circuits and Systems (ISCAS'99), Orlando, Florida, USA, 1, 383-386, May, 1999.

[10] Fisher, L. T.,"Unateness properties of AND-ExclusiveOR logic circuits", IEEE Trans. Comput., 23(2), 166-172, Feb., 1974.

[11] Green, D. H. (1991). "Families of Reed-Muller canonical forms", Int. J. Electronics, 70, 259-280.

[12] Marek-Sadowska, M., Guner, G., Krishnamurthy, T., Gargeshwari, S. and Sharma, Ch., "Minimized Generalized Partially Mixed Polarity Reed-Muller Expansion", In: Proc. 3rd Int. Workshop on Applications of the ReedMuller Expansion in Circuit Design (Reed-Muller'97), Oxford, United Kingdom, pp. 35-44, Sep., 1997.

[13] Perkowski, M. A. and Chrzanowska-Jeske, M., "An exact algorithm to minimize mixed-radix exclusive sums of products for incompletely specified Boolean functions", In: Proc. IEEE Int. Symp. on Circuits and Systems (ISCAS'90), New Orleans, Louisiana, USA, pp. 16521655, May, 1990.

[14] Reddy, S. M., "Easily testable realizations for logic functions", IEEE Trans. Comput., 21(11), 1183-1188, Nov., 1972.

[15] Sarabi, A. and Perkowski, M. A., "Fast exact and quasiminimal minimization of highly testable fixed-polarity AND/XOR canonical networks", In: Proc. 29th ACM/ IEEE Design Automation Conf., pp. 30-35, Jun., 1992.

[16] Sasao, T. and Fujita, M. Eds., Representations of Discrete Functions. Kluwer Academic Publisher, Boston, 1996.

[17] Song, N. and Perkowski, M. A., "Minimization of exclusive sum-of-products expressions for multiple-valued input, incompletely specified functions", IEEE Tran. on Computer-Aided Design of Integrated Circuits and Systems, 15(4), 385-395, Apr., 1996.

[18] Tsai, C. C. and Sadowska, M. (1994) "Minimization of fixed polarity AND/XOR canonical network", IEE Proc., Comput. Digit. Tech., 141(6), 369-374.

[19] Wu, H., Perkowski, M. A., Zeng, X. and Zhuang, N., "Generalized partially-mixed-polarity Reed-Muller expansion and its fast computation", IEEE Trans. on Comput., 45(9), 1084-1088, Sep., 1996.

[20] Zeng, X., Perkowski, M. A., Wu, H. and Sarabi, A., "A new exact algorithm for highly testable generalized partially-mixed-polarity Reed-Muller forms", In: Proc. IFIP WG10.5 Workshop on Applications of the ReedMuller Expansion in Circuit Design, Makuhari, Chiba, Japan, pp. 231-239, Aug., 1995.

\section{Authors' Biographies}

Bogdan J. Falkowski received the MSEE degree from Technical University of Warsaw, Poland and the Ph.D. degree in Electrical and Computer Engineering from Portland State University, Oregon, USA. His industrial experience includes research and development positions at several companies. He then joined the Electrical and Computer Engineering Department at Portland State University. Since 1992 he has been with the School of Electrical and Electronic Engineering, Nanyang Technological University in Singapore where he is currently an Associate Professor. His research interests include VLSI systems and design, switching circuits, testing, and design of algorithms. He specializes in the design of digital circuits with the use of spectral methods and has published 3 book chapters and over 130 refereed journal and conference articles in this area. He is a senior member of the IEEE, member of international advisory committee for International Conference on Applications of Computer Systems, guest editor of VLSI Design journal, and technical chair for IEEE International Conference on Information, Communication and Signal Processing held in December, 1999 in Singapore.

Chip-Hong Chang received the BEng degree from National University of Singapore, and the M.Eng. and Ph.D. degrees in Electrical and Electronic Engineering from Nanyang Technological University, Singapore. $\mathrm{He}$ is currently an Assistant Professor at the School of Electrical and Electronic Engineering, Nanyang Technological University. His research interests include optimization and synthesis of VLSI digital circuits, graph theory and symbolic algorithms, and formal verification. He has published over 30 refereed journal and conference articles in these areas. $\mathrm{He}$ is a member of the IEEE. 

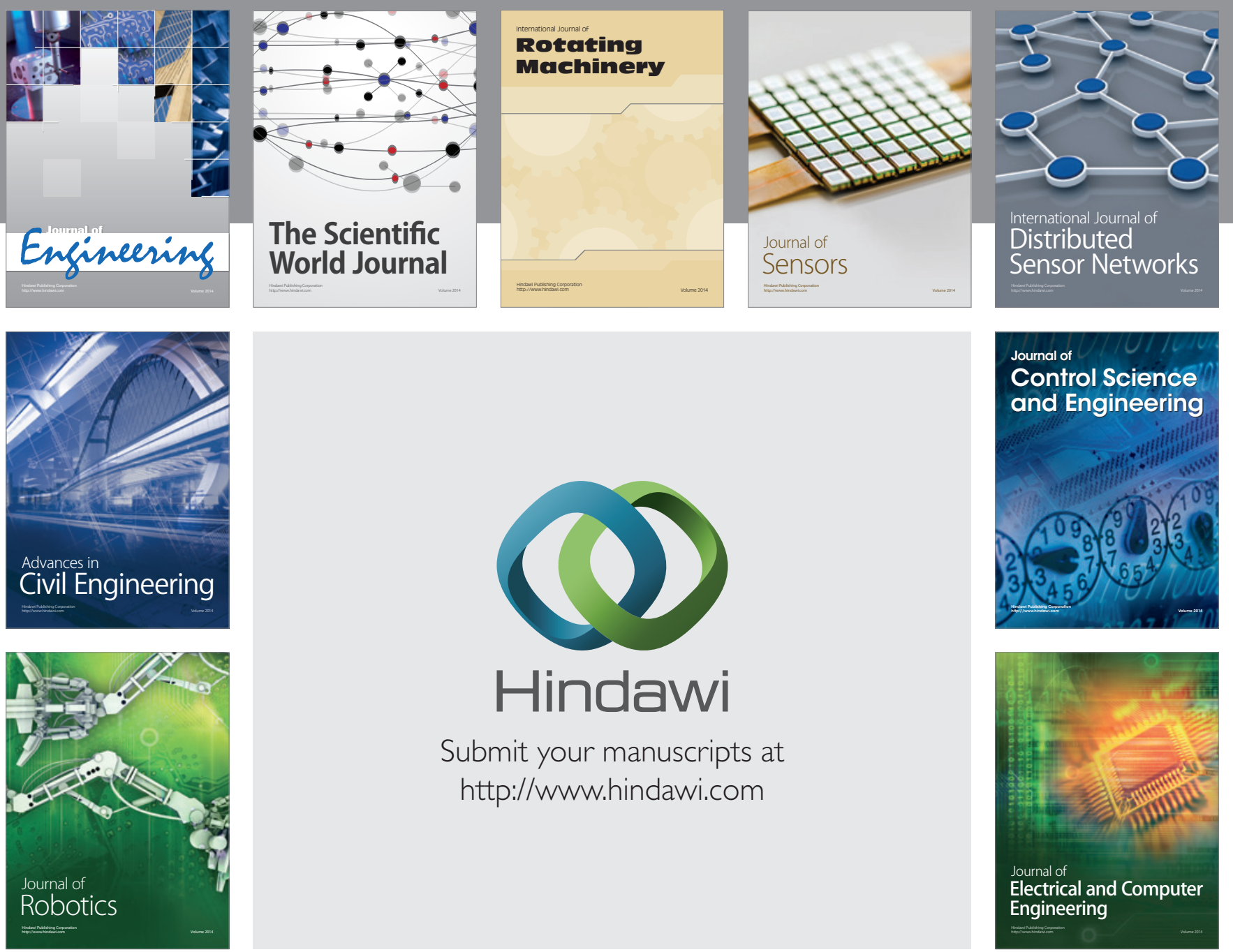

Submit your manuscripts at

http://www.hindawi.com
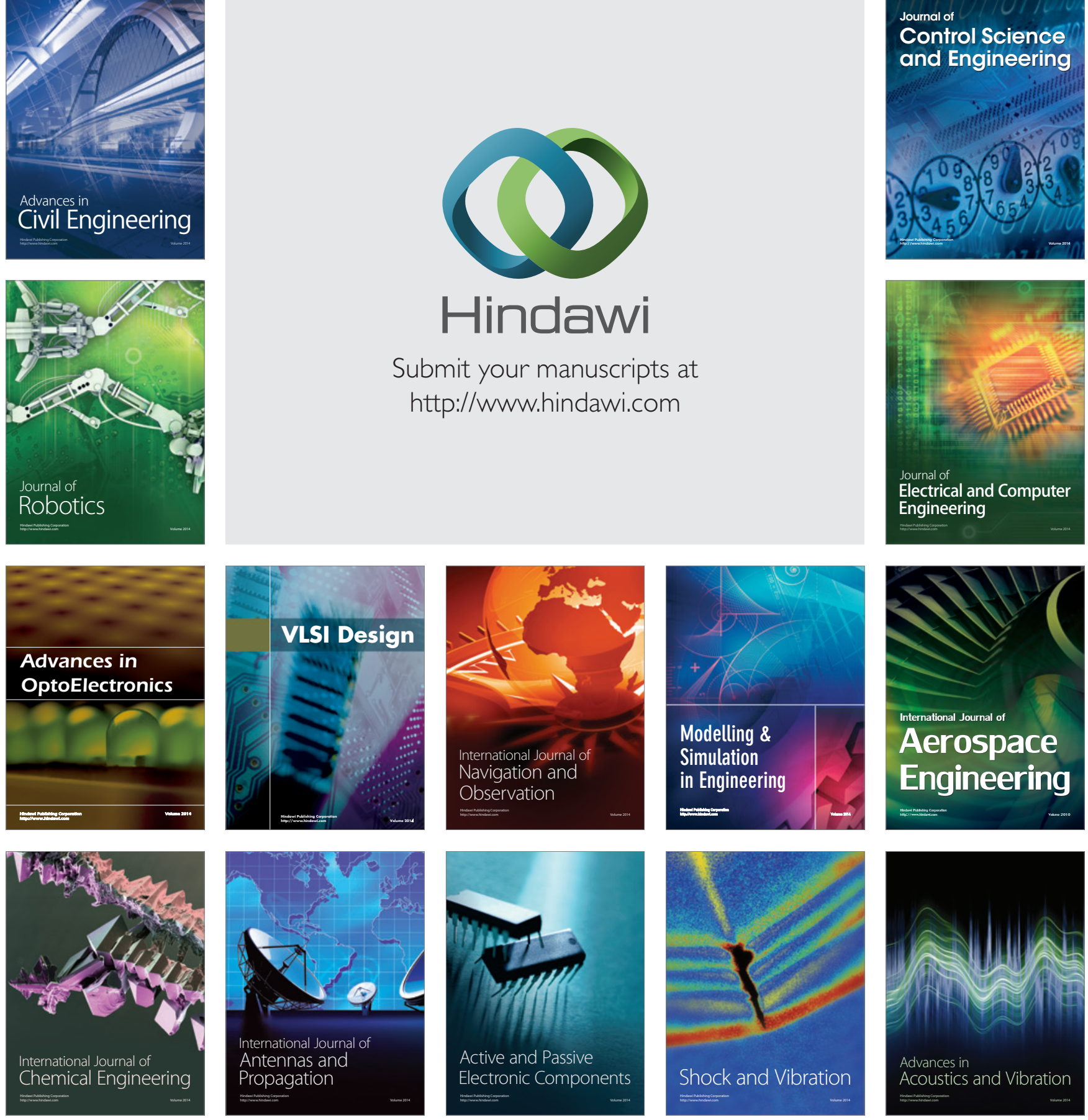